\title{
Surgical management of a brain abscess due to plant foreign body in a dog
}

\author{
Ana Cloquell ${ }^{1}$ and Isidro Mateo ${ }^{1,2 *}$ \\ ${ }^{1}$ Veterinary Teaching Hospital, Alfonso X el Sabio University, Madrid, Spain \\ ${ }^{2}$ VETSIA Veterinary Hospital, Madrid, Spain
}

\begin{abstract}
Background: Intracranial abscesses as a result of grass awn migration have been rarely described in the veterinary literature. The identification of their radiological features is mandatory for proper diagnosis. As occurs with abscesses in other organs, surgical drainage and directed antibiotic therapy should be considered the treatment of choice.

Case Description: A clinical case of a Great Dane dog with forebrain signs and magnetic resonance imaging and computed tomography findings compatible with intracranial abscess associated with inflammatory changes in orbital musculature is described. An exploratory rostro-tentorial craniotomy with durotomy was performed, allowing the drainage of purulent content and the extraction of a plant foreign body from the cerebral parenchyma. Antibiotic treatment was instituted and the patient was discharged without recurrence of neurological deficits other than quarterly seizures. Six months later, revision magnetic resonance was performed, revealing the resolution of the intracranial lesion and the normalization of the extracranial tissues.

Conclusion: This is the first case in veterinary literature in which a grass awn has been surgically extracted from the brain of a dog with long-term outcome described. Observed changes in the extracranial musculature were fundamental to establish the pre-surgical diagnosis of a migratory foreign body.

Keywords: Brain abscess, Plant foreign body, Rostro-tentorial craniectomy.
\end{abstract}

\section{Introduction}

Brain abscessation is a rare diagnosis in dogs. It is an accumulation of purulent content inside the cerebral parenchyma usually associated with the extension of extracranial infections, migration of foreign bodies, or bites (Burgisser and Hintermann, 1967; McCandlish and Ormerod, 1978; Seiler et al., 2001; Dennis et al., 2005; Sturges et al., 2006; Mateo et al., 2007; Bilderback and Faissler, 2009). Even more infrequently its presence has been described as a result of the hematogenous spread of a distant infection (e.g., bacterial endocarditis), or as a complication in immunosuppressed patients (Smith et al., 2007). In cats, brain abscesses have usually been described as a complication of middle ear infections or bite wounds (Klopp et al., 2000; Sturges et al., 2006; Costanzo et al., 2011). Infections caused by plant material (particularly, grass awns) are a common cause of consultation in dogs. Grass awns are especially good at migrating through the soft tissue since their morphology facilitates penetration and progressive advancement (Brennan and Ihrke, 1983). Grass awns usually enter the head through the skin, the ear canal, the conjunctiva, the paranasal sinuses, or the oral cavity (Burgisser and Hintermann, 1967; McCandlish and Ormerod, 1978; Dennis et al., 2005; Mateo et al., 2007). The diagnosis of a brain abscess is based on advanced imaging, usually computerized tomography (CT) or magnetic resonance imaging (MRI)
(Klopp et al., 2000; Seiler et al., 2001; Sturges et al., 2006; Mateo et al., 2007; Smith et al, 2007; Bilderback and Faissler, 2009; Costanzo et al., 2011). However, findings are not pathognomonic of brain abscesses and may be compatible with other brain lesions such as primary tumors, metastases, infarcts, granulomas, or hematomas in resolution (Thomas, 1999). Definitive diagnosis requires the combination of advanced imaging, cerebrospinal fluid (CSF) analysis, and aerobic and anaerobic cultures (Tipold, 1995). Previous medical history (bites, trauma, etc.,) and findings in the physical examination may restrict differential diagnosis (Klopp et al., 2000; Sturges et al., 2006; Bilderback and Faissler, 2009; Costanzo et al., 2011). At present, the benefits of medical treatment over surgery treatment, or vice versa, are not demonstrated in the veterinary literature. However, a good prognosis has been described in patients in which a surgical resolution of the lesion has been pursued (Sturges et al., 2006; Bilderback and Faissler, 2009; Costanzo et al., 2011). Nevertheless, all surgically treated brain abscesses described in veterinary literature were produced by bites or disseminated infections from extracranial tissues (Sturges et al., 2006; Bilderback and Faissler, 2009; Bentley et al., 2011; 2015; Costanzo et al., 2011). This case represents the first clinical description of an intracranial abscess produced by the migration of a plant foreign body which was surgically treated with long-term radiological follow-up. 


\section{Case Details}

A 3-year-old female Great Dane was referred for acute onset behavioral changes (aggression and disorientation) of 2 weeks duration, and seizures from 24 hours prior to presentation. Physical examination revealed no abnormalities in body temperature, body condition score, heart rate, pulse quality, or cardiopulmonary auscultation. All lymph nodes were considered of normal size and no pain was elicited upon manipulation of the orbital, aural, or cranial musculature. Neither was there any history of dermatological or traumatic lesions in the eye, ear, or mouth. The neurological examination revealed the patient to be obtunded with decreased postural reactions and menace response worse in the left side. Neuroanatomic lesion localization was considered focal in the right prosencephalon with a possible increase in intracranial pressure. Hematological and serum biochemical analysis showed no abnormalities. Magnetic resonance imaging was performed using a low-field magnet (Airis Mate; Hitachi, Tokio, Japan). The study revealed a left-sided single frontal intra-axial lesion affecting gray and white matter adjacent to the internal capsule at the level of the optic canal (Fig. 1). The lesion presented signal homogeneity characterized by hyperintensity on T2W and FLAIR sequences and hypointensity in T1W sequences with peripheral enhancement after intravenous contrast administration (Fig. 1A and 1B). The lesion had a round shape with the greatest diameter of $2.84 \mathrm{~cm}$. This enhancement was also recognized in the surrounding meninges following the brain surface following sulci. A small linear tract observed was directed dorsally from the lesion through the corona radiata (Fig. 1B). The lesion was associated with extensive perilesional vasogenic edema that worsened the mass effect characterized by a midline shift. The orbital and medial pterygoid muscles also showed an intense enhancement (Fig. 2). The wing of the presphenoid bone was thinner than the contralateral bone (Fig. 1B). A CT scan (Astelion 16, Toshiba, Tokio, Japan) was performed after MRI that allowed the identification of osteolysis in the presphenoid bone immediately dorsal to the orbital fissure (Fig. 1C). CT scan also revealed a hypoattenuating intra-axial lesion located in the left frontoparietal lobe together with diffuse hypoattenuation of tissues adjacent to the left optic nerve and the left pterygoid muscle. Finally, CSF analysis showed no abnormalities. On the basis of advanced radiological findings, the main differential diagnosis was an intracranial abscess due to the extension of the extracranial septic process through presphenoid bone. Because of rapid neurologic deterioration and suspected increased intracranial pressure, surgical excision was planned. A modified left rostrotentorial craniectomy was performed. Temporalis muscle and fascia were retracted, extending the incision cranially through the temporal line. Once the frontal and presphenoid bones were exposed an area of osteolysis was observed in the wing of the presphenoid bone immediately dorsal to the orbital fissure. The craniectomy (approximately 3 $\mathrm{cm}$ diameter) was centered on the osseous defect. A mucopurulent discharge presented immediately after the incision of the dura mater. A sample was collected for its microbiological study. The surgical site was flushed with sterile saline revealing the tip of a grass awn which was extracted from the brain parenchyma together with a small amount of purulent fluid (Fig. 3). After extensive flushing, the meninges were left open to allow drainage and the incision was closed as per routinely. Postoperative CT showed the correct location of the craniectomy and resolution of the abscess which was substituted by gaseous content but remained inside and on the dorsal surface of the frontal lobe (Fig. 4). Immediate postoperative therapy consisted of amoxicillin + clavulanic acid (Synulox 500, Zoetis Spain SL) 22 mg/kg PO, q8h; marbofloxacin (Marbocyl P80, Vetoquinol SA) $5 \mathrm{mg} / \mathrm{kg}$ PO, q24h; metronidazole (Flagyl 250, Sanofi-aventis SA) $15 \mathrm{mg} / \mathrm{kg}$ PO, q12h; prednisone (Prednisona Kern Pharma 30mg, Kern Pharma) $0.5 \mathrm{mg} / \mathrm{kg} \mathrm{PO}$, q12h; tramadol (Tramadol

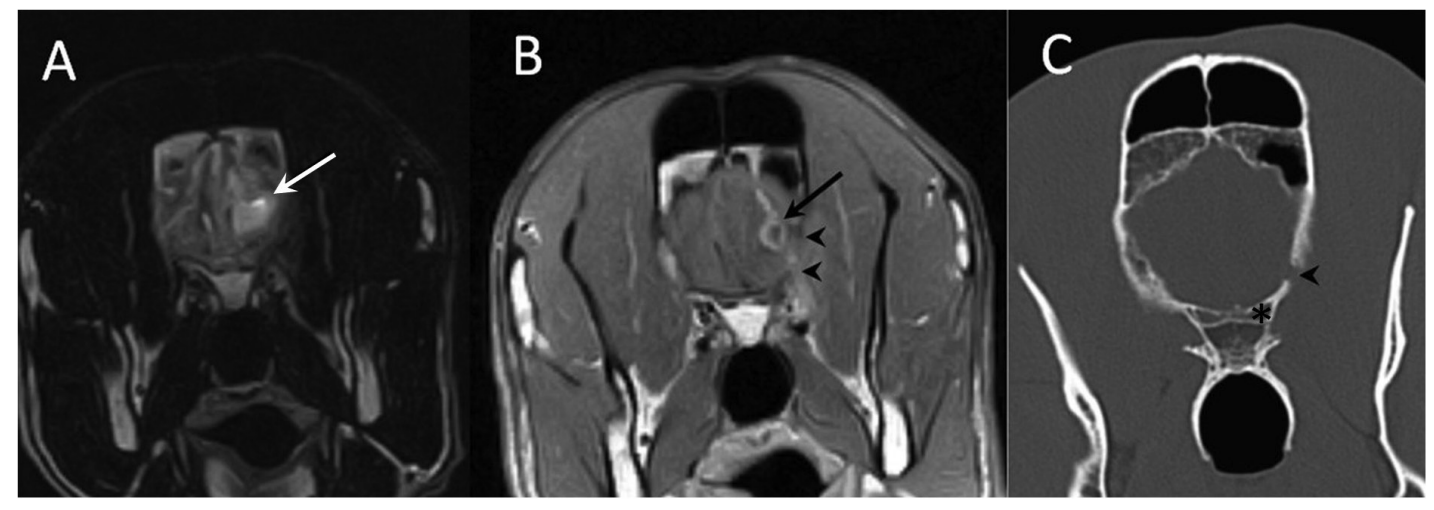

Fig. 1. (A) Transverse T2-weighted image, (B) transverse post-contrast $T 1$-weighted image, and (C) transverse CT image obtained at the level of the optic canal (asterisk). The lesion can be seen as a hyperintense (arrow in A) and ring-enhancing mass (arrow in B) located in the left frontal lobe. Note thinning of the wing of the presphenoid bone (arrowheads in B and C). 


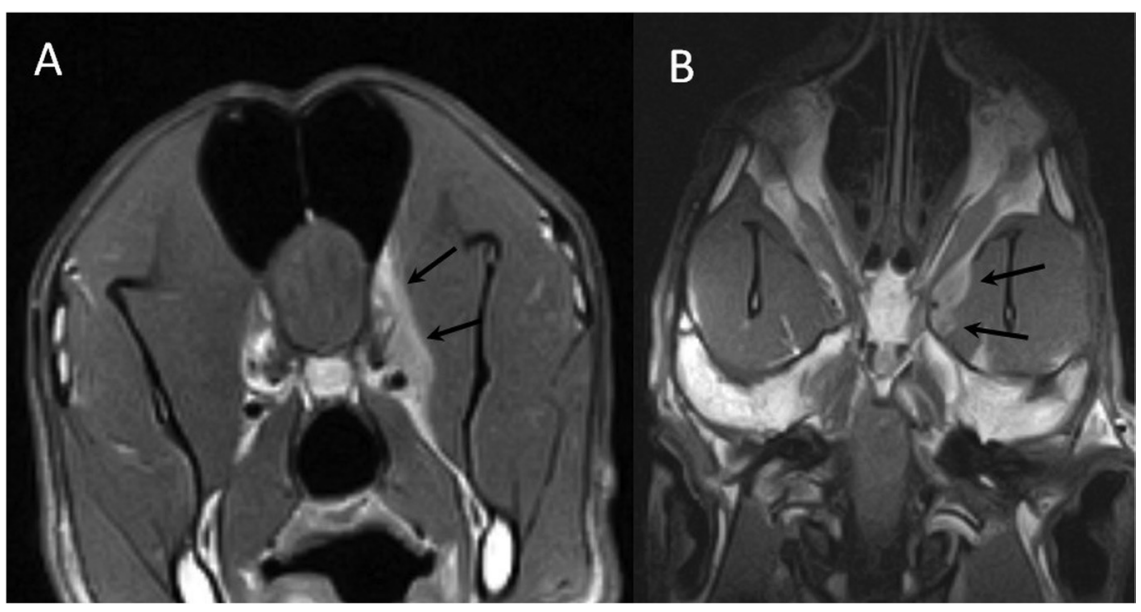

Fig. 2. (A) Transverse and (B) dorsal post-contrast T1-weighted images revealing enhancement of orbital musculature and medial pterygoid muscle (arrows).

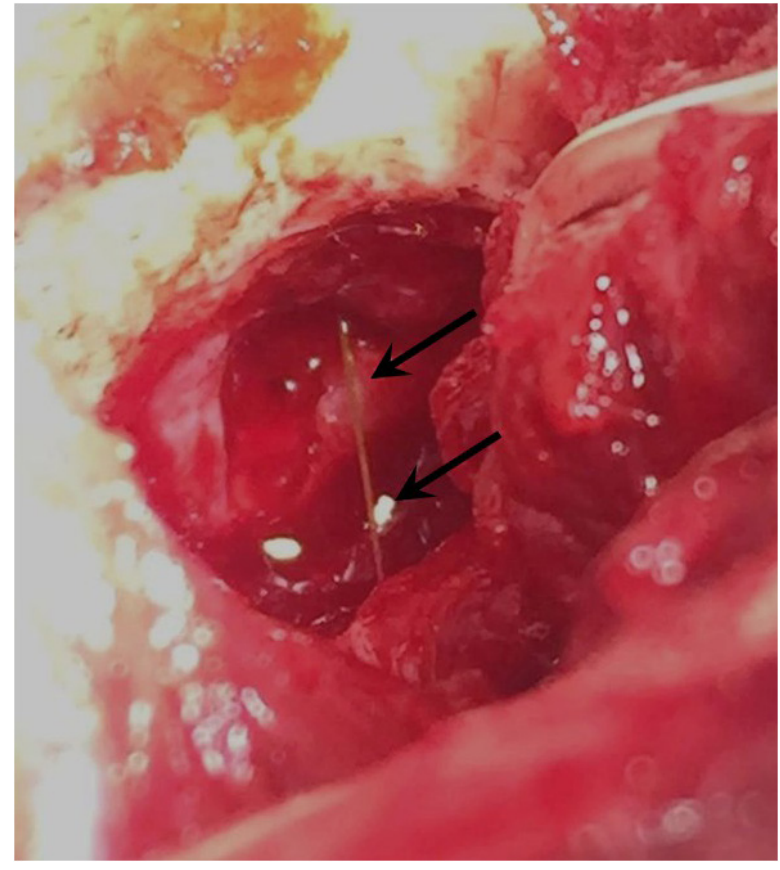

Fig. 3. Intraoperative view after left rostrotentorial craniectomy and durotomy above the orbital fissure. Note durectomy and the presence of a grass awn (arrows) overlying brain parenchyma once extracted from the frontal lobe.

retard Combix $100 \mathrm{mg}$, Laboratorios Combix S.L.U) $2 \mathrm{mg} / \mathrm{kg}$ PO, q8h; famotidine (Famotidina Normon 40 $\mathrm{mg}$; Laboratorios Normon SA) $0.7 \mathrm{mg} / \mathrm{kg}$ PO, q24h; and phenobarbital (Phenoleptil $100 \mathrm{mg}$, Le Vet BV) 2.5 $\mathrm{mg} / \mathrm{kg}$ PO, q12h. Cultured samples were positive for Pseudomonas aeruginosa sensitive to ciprofloxacin, imipenem, and amikacin among other antibiotics. Based on these results, antibiotic therapy was changed to ciprofloxacin (Ciprofloxacino 500, Laboratorios Normon SA) $10 \mathrm{mg} / \mathrm{kg}$ PO, q12h and metronidazole 15 $\mathrm{mg} / \mathrm{kg}$ PO, q12h for 2 months. Prednisone, tramadol, and famotidine were withdrawn and phenobarbital was maintained at $2.5 \mathrm{mg} / \mathrm{kg}$ q12h. Six-month postoperative revision magnetic resonance revealed no signs suggesting the existence of an inflammatory process (Fig. 5). In the area where the abscess had been located, a fluid-filled cavity suggesting being CSF was observed. One year follow-up revealed the patient had seizures every 3 months with neurological examination remaining normal. The patient died 2 years later of unrelated causes.

\section{Discussion}

Most of the brain abscesses diagnosed in veterinary literature are produced by direct extension of extracranial infections such as otitis media/interna, rhinitis, exposed trauma, or foreign body migration (Burgisser and Hintermann, 1967; McCandlish and Ormerod, 1978; Klopp et al., 2000; Seiler et al., 2001; Dennis et al., 2005; Sturges et al., 2006; Mateo et al., 2007; Bilderback and Faissler, 2009; Costanzo et al., 2011). MRI and CT are very effective methods for the detection of brain abscesses in both dogs and cats (Thomas, 1999). Classic MRI findings consist of the presence of a ring enhanced lesion with a hypointense central area in $\mathrm{T} 1 \mathrm{~W}$ images and hyperintense in $\mathrm{T} 2 \mathrm{~W}$ images associated with perilesional edema and variable mass effect (Thomas, 1999; Klopp et al., 2000; Costanzo et al., 2011). An inflammatory granuloma associated with plant material has also been described in the brainstem of a dog (Mateo et al., 2007). The CT findings consist of a hypoattenuating lesion surrounded by an intense and ring-shaped enhancement (Bilderback and Faissler, 2009). These findings, both MRI and CT, are not specific. With such findings, differential diagnoses must include neoplastic and vascular (hemorrhagic infarction) diseases. However, when inflammatory changes in extra-cranial tissue are observed, differential diagnosis narrows; with infectious dissemination being almost the only possibility (Klopp et al., 2000; Sturges 


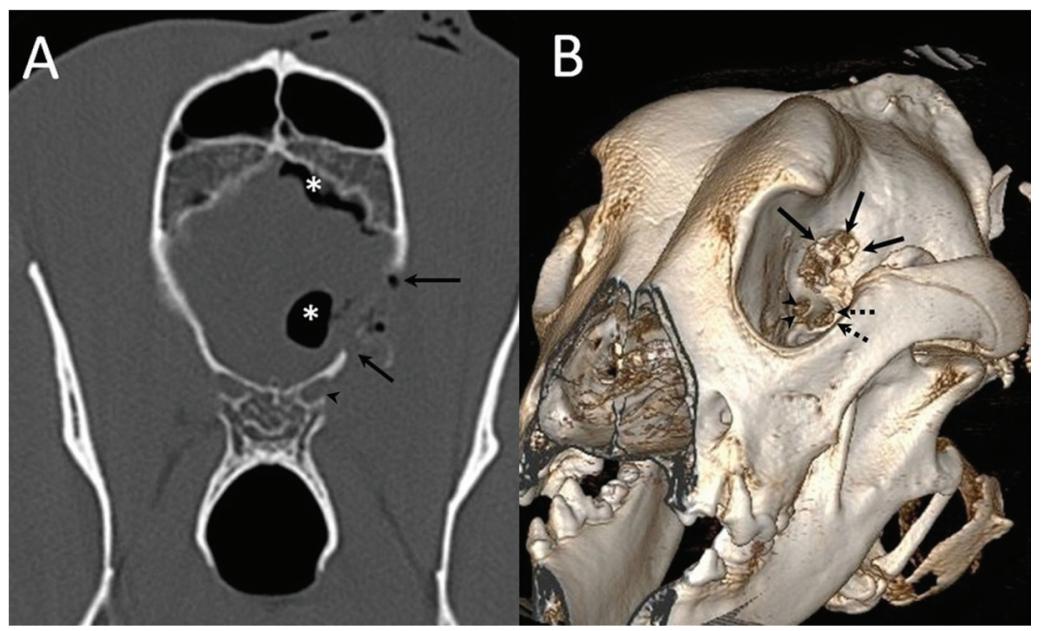

Fig. 4. (A) Transverse and (B) 3D reconstruction postoperative CT images. Note increased defect on the presphenoid bone (arrows) immediately dorsal to the optic canal (arrowhead) and orbital fissure (dotted arrow). Also, note the presence of hypoattenuating content inside and over the dorsal surface of the frontal lobe (asterisks) compatible with air.

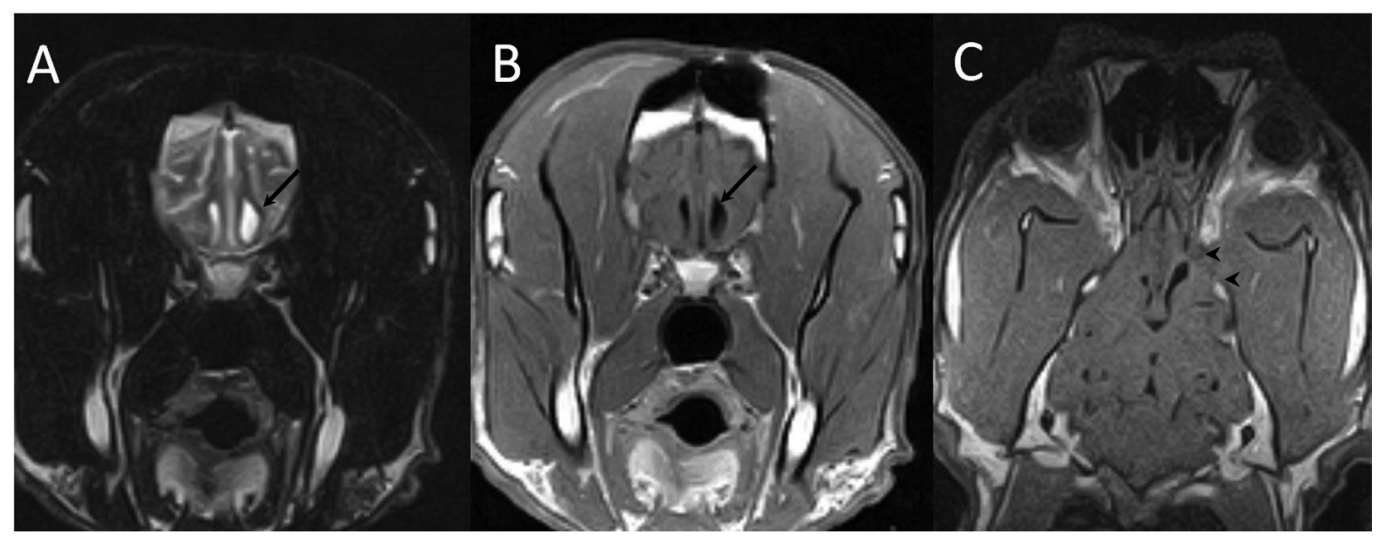

Fig. 5. (A) Transverse T2-weighted, (B) transverse post-contrast T1-weighted image, and (C) dorsal postcontrast T1-weighted image obtained 6 months after surgery. Note the absence of any contrast enhancement and residual lesion connecting the lateral ventricle (arrows). Also, note the defect in the presphenoid bone (arrowheads in C).

et al., 2006, Bilderback and Faissler, 2009; Costanzo et al., 2011). For this reason, when a lesion with these described features is observed, an attempt should be made to identify changes in adjacent tissue which would suggest foreign body migration or dissemination of an infectious process rather than glioma or hemorrhage. The case presented here did not reveal any previous history of ocular or oral disease nor any clinical sign suggestive of extracranial involvement. In human medicine, brain abscesses usually occur a few days after the entry of the infectious agent into the CNS; however, there is considerable variation (Hakan et al., 2006). Moreover, not all materials have the same possibility of abscess formation, e.g., wooden foreign bodies cause abscess formation in $48 \%$ of cases (Miller et al., 1977) and foreign metallic bodies have a lower frequency for abscess formation (10\%-33\%). The latter are often hot and sharp at penetration and perhaps produce a relatively sterile wound (Hagan, 1971). In the presence of an infectious process in the CNS, it would be expected to find an increased cell count in CSF, usually neutrophilic pleocytosis, which is the hallmark of bacterial infections of the CNS (Tipold, 1995). However, in this case, the CSF analysis was normal. This could be due to the intra-axial location of the abscess without dissemination on the subarachnoid space. If a cerebral abscess is suspected, a culture of the CSF can be performed. However, a negative result does not exclude a diagnosis, particularly if the patient has been previously treated with antibiotics (Radaelli and Platt, 2002). Antibiotic therapy remains the most important issue in the treatment of intracranial 
abscesses and should be orientated, ideally, by the results of the microbiological cultures obtained during the surgical procedure (Bilderback and Faissler, 2009; Costanzo et al., 2011). Abscesses are usually caused by aerobic or anaerobic bacteria, but sometimes they can be polymicrobial or fungal (Bentley et al., 2011; 2015). In the absence of positive cultures, some studies in human medicine recommend a combination of broad-spectrum intravenous antimicrobial therapy combining the third-generation cephalosporins (for Gram-positive and Gram-negative), metronidazole (for anaerobes), and ampicillin (to additionally cover other Gram-positive) (Sjölin et al., 1993; Nau et al., 1998; Lu et al., 2006). Usually, intravenous antibiotic therapy is established for 4-8 weeks followed by control CT before starting oral antibiotic treatment (Lu et al., 2006). In the veterinary literature, it has been recommended to maintain intravenous therapy until clinical improvement is observed. Oral medication should be maintained until at least 10-14 days after the resolution of the clinical signs (Bilderback and Faissler, 2009).

The administration of anti-inflammatory doses of corticosteroids together with antimicrobials is associated with a better outcome (fewer sequelae and mortality) in human patients with bacterial meningoencephalitis (van de Beek et al., 2004). Corticosteroids may reduce adverse effects produced during antibiotic-induced bacterial lysis. Endotoxins, peptidoglycans, and fragments of the cell wall can release pro-inflammatory cytokines that can induce profound meningeal inflammation and brain edema (Nau et al., 1998). The timing of administration is critical to prevent the release of inflammatory mediators secondary to bacterial lysis. In humans, doses of dexamethasone $(0.1-0.15 \mathrm{mg} / \mathrm{kg}$, IV, q4 hours, for 2-4 days) are recommended before, or at the same time, as the first dose of antimicrobials (van de Beek et al., 2004). However, its usefulness in patients with abscesses of bacterial origin has not been demonstrated with higher rates of mortality and morbidity (Nau et al., 1998; Hakan et al., 2006; Lu et al., 2006).

All the previously described cases of dogs with brain abscess or granuloma associated with plant foreign body were diagnosed postmortem (Burgisser and Hintermann, 1967; McCandlish and Ormerod, 1978; Dennis et al., 2005; Mateo et al., 2007). A poor prognosis has also been associated with bacterial meningoencephalitis (Radaelli and Platt, 2002). However, cases (dogs and cats) with brain abscesses due to bites or extension of otogenic infections seem to have a much more favorable prognosis, particularly after surgical treatment (Bilderback and Faissler, 2009; Costanzo et al., 2011). Surgery has been advised in patients with increased intracranial pressure due to the mass effect, or when sample collection is required for microbiological study (Bilderback and Faissler, 2009; Costanzo et al., 2011). Surgery will also allow drainage of the lesion and collection of samples for culture, thus guiding the most appropriate antibiotic regimen. Nevertheless, the effect of surgery on prognosis has not been established.

This case represents the only case in which long term (6 months) radiological follow-up of the lesion was carried out by means of MRI, proving the disappearance of the lesion. The seizures that the patient had during the rest of its life were probably due to epileptogenic focus in the cerebral parenchyma where the abscess was located.

In conclusion, we can highlight the typical radiological features of brain abscesses consisting of an intra-axial lesion with intense ring enhancement (both in CT and MRI). If an extracranial abnormality is found in adjacent tissues, the diagnosis of a cerebral abscess is almost definitive. Surgical treatment that allows decompression, drainage, lavaging, and collection of samples for cytological and microbiological examination should be considered in patients with brain abscesses.

\section{References}

Bentley, R.T., Faissler, D. and Sutherland-Smith, J. 2011. Successful management of an intracranial phaeohyphomycotic fungal granuloma in a dog. J. Am. Vet. Med. Assoc. 239, 480-485.

Bentley, R.T., Heng, H.G., Thompson, C., Lee, C.S., Kroll, R.A., Roy, M.E., Marini, L., Heo, J. and Wigle, W.L. 2015. Magnetic resonance imaging features and outcome for solitary central nervous system coccidioides granulomas in 11 dogs and cats. Vet. Radiol. Ultrasound 56, 520-530.

Bilderback, A.L. and Faissler, D. 2009. Surgical management of a canine intracranial abscess due to a bite wound. J. Vet. Emerg. Crit. Care 19, 507-512.

Brennan, K.E. and Ihrke, P.J. 1983. Grass awn migration in dogs and cats: a retrospective study of 182 cases. J. Am. Vet. Med. Assoc. 182, 1201-1204.

Burgisser, H. and Hintermann, J. 1967. Cerebral damages due to foreign bodies from the nasal cavity in the dog. Schweiz. Arch. Tierheilkd. 10, 537-538.

Costanzo, C., Garosi, L.S., Glass, E.N., Rusbridge, C., Stalin, C.E. and Volk, H.A. 2011. Brain abscess in seven cats due to a bite wound: MRI findings, surgical management and outcome. J. Feline Med. Surg. 13, 672-680.

Dennis, M.M., Pearce, L.K., Norrdin, R.W. and Ehrhart, E.J. 2005. Bacterial Meningoencephalitis and ventriculitis due to migrating plant foreign bodies in three dogs. Vet. Pathol. 42, 840-844.

Hagan, R.E. 1971. Early complications following penetrating wounds of the brain. J. Neurosurg. 34, $132-141$. 
Hakan, T., Ceran, N., Erdem, I., Berkman, M.Z. and Göktaş, P. 2006. Bacterial brain abscesses: an evaluation of 96 cases. J. Infect. 52, 359-366.

Klopp, L.S., Hathcock, J.T. and Sorjonen, D.C. 2000. Magnetic resonance imaging features of brain stem abscessation in two cats. Vet. Radiol. Ultrasound 41, 300-307.

Lu, C.H., Chang, W.N. and Lui, C.C. 2006. Strategies for the management of bacterial brain abscess. J. Clin. Neurosci. 13, 979-985.

Mateo, I., Lorenzo, V., Muñoz, A. and Pumarola, M. 2007. Brainstem abscess due to plant foreign body in a dog. J. Vet. Intern. Med. 21, 535-538.

McCandlish, I.A.P. and Ormerod, E.J. 1978. Brain abscess associated with a penetrating foreign body. Vet. Rec. 102, 380-381.

Miller, C.F., Brodkey, J.S. and Colombi, B.J. 1977. The danger of intracranial wood. Surg. Neurol. 7, 95-103.

Nau, R., Sörgel, F. and Prange, H.W. 1998. Pharmacokinetic optimization of the treatment of bacterial central nervous system infections. Clin. Pharmacokinet. 35, 223-246.

Radaelli, S.T. and Platt, S.R. 2002. Bacterial meningoencephalomyelitis in dogs: a retrospective study of 23 cases (1990-1999). J. Vet. Intern. Med. $16,159-163$.
Seiler, G., Cizinauskas, S., Scheidegger, J. and Lang, J. 2001. Low-field magnetic resonance imaging of a pyocephalus and a suspected brain abscess in a german sherperd dog. Vet. Radiol. Ultrasound 42, 417-422.

Sjölin, J., Lilja, A., Eriksson, N., Arneborn, P. and Cars, O. 1993. Use of cefotaxime and metronidazole for treating cerebral abscesses. Clin. Infect. Dis. 17, 857-863.

Smith, P.M., Haughland, S.P. and Jeffery, N.D. 2007. Brain abscess in a dog immunosuppressed using cyclosporin. Vet. J. 173, 675-678.

Sturges, B.K., Dickinson, P.J., Kortz, G.D., Berry, W.L., Vernau, K.M., Wisner, E.R. and LeCouteur, R.A. 2006. Clinical signs, magnetic resonance imaging features, and outcome after surgical and medical treatment of otogenic intracranial infection in 11 cats and 4 dogs. J. Vet. Intern. Med. 20, 648-656.

Thomas, W.B. 1999. Nonneoplastic disorders of the brain. Clin. Tech. Small Anim. Pract. 14, 125-147.

Tipold, A. 1995. Diagnosis of inflammatory and infectious diseases of the central nervous system in dogs: a retrospective study. J. Vet. Intern. Med. 9, 304-314.

van de Beek, D., de Gans, J., McIntyre, P. and Prasad, K. 2004. Steroids in adults with acute bacterial meningitis: a systematic review. Lancet Infect. Dis. 4, 139-143. 